\title{
FDA expedited approval and implications for rational formulary and health plan design
}

\author{
Erin A Ferries, PhD, MPH; William K Fleming, PharmD; and William H Shrank, MD, MSHS
}

There are few times in recent history where the pharmaceutical pipeline has generated such anticipation for diseases with unmet needs. The volume of gene, specialty, and breakthrough therapies receiving US Food and Drug Administration (FDA) approval has reached unprecedented levels and is expected to grow significantly in the coming years. Even with the FDA granting a record number of approvals, every day that a drug spends in the discovery process represents precious moments for patients with degenerative or life-threatening diseases.

In response to increased pressure on the FDA to make therapies available to patients sooner, the agency has developed 4 regulatory pathways intended to expedite the approval process for drugs indicated for conditions with high, unmet need: fast-track designation, breakthrough therapy designation, accelerated approval, and priority review designation. ${ }^{1}$

The establishment of the FDA expedited pathways has resulted in drug approvals occurring at record speed, with $73 \%$ and $60 \%$ of novel drugs in 2018 and 2019, respectively, receiving an expedited approval., ${ }^{2,3}$ The FDA prioritization of certain approvals and expedited pathways are warranted for drugs with potentially life-saving properties or those anticipated to offer significant advancement in medical care, even if it means greater risk for unknown side effects and potentially even failure in the real-world setting. While these are well-intended policies, there are sometimes unintended consequences.

One consequence of medical innovation is the often considerable price tag. Among groundbreaking gene therapies, for example, treatment costs are estimated to range from hundreds of thousands of dollars to $\$ 2.1$ million, in the case of a single round of Novartis's Zolgensma. ${ }^{4-6}$ While several therapies are indicated for rare diseases, use among even a few thousand patients could have an extraordinary financial impact on patients, Medicare health plan sponsors, and the health care system overall.

The influx and rate of new-tomarket, high-cost drugs in the health care marketplace is a major contributing factor to the skyrocketing costs of prescription drugs in America. As of 2020, specialty drugs represent approximately $50 \%$ of total drug spend and is expected to grow, considering that nearly $80 \%$ of new drug approvals are categorized as specialty. ${ }^{2,7}$ While the expedited approval process does offer a mechanism to bring additional competition to the drug marketplace more rapidly, it also means that more drugs are coming to market based on less established evidence.

In 2018 , over $40 \%$ of novel drugs were approved based on the results of only 1 clinical trial, or preliminary data from phase 1 or 2 trials, with no

\begin{abstract}
Author affiliations
Erin A Ferries, PhD, MPH; William K

Fleming, PharmD; and William H Shrank, MD, MSHS, Humana, Inc., Louisville, KY.

AUTHOR CORRESPONDENCE:

Erin A Ferries, 608.633.1743;

eferries@humana.com

J Manag Care Spec Pharm. 2021;27(5):682-84

Copyright $\odot 2021$, Academy of Managed Care Pharmacy. All rights reserved.
\end{abstract}

phase 3 supporting evidence. ${ }^{8}$ Many other studies were reliant on surrogate endpoints that were not designed to assess outcomes for broad patient populations or understanding of longterm effects. This dynamic leaves health plans scrambling to prepare for the potential implications of an FDA approval, given that plans, along with patients, may end up paying for a treatment that might never prove to be effective.

While many drugs receive expedited approval with the requirement that sponsors conduct additional confirmatory studies, health plans and pharmacy benefit sponsors are saddled with paying the full price for these drugs shortly after FDA approval. Meanwhile, drug manufacturers 
profit from extraordinary prices, despite limited proof of effectiveness. For example, in 2016, Eli Lilly's cancer drug (Lartruvo) was granted accelerated approval and breakthrough designation by the FDA after completion of a single phase 2 randomized clinical trial conducted with 133 patients. ${ }^{9}$ In 2019, this drug was withdrawn from the market after failing to improve patient survival rates. Still, the drug manufacturer earned an estimated profit of over $\$ 500$ million in the 2 years that the drug was on the market.

With the surge of ultra high-cost drugs gaining approval, the contemporary formulary is a clinical tool that enables health plans to evaluate the clinical appropriateness and safety of treatment options for their unique patient populations. Formularies also function as cost-containment tools to shield patients and payers from unnecessary health care costs and low value care, while driving the use of more affordable, clinically equivalent therapeutic options.

In some cases, mandatory drug coverage laws demand that certain classes of drugs must be covered by health plans. When drugs that fall into one of these required coverage categories receive expedited approval based on limited efficacy data, they receive unconditional coverage and related payment, regardless of their demonstrated real-world effectiveness or the lack thereof. For example, Inrebiq, an antineoplastic used to treat certain types of myelofibrosis, received priority review and orphan drug and protected status in 2019, although it had similar efficacy to the myelofibrosis drug Jakafi, while also presenting a potentially higher toxicity profile and higher costs. ${ }^{10,11}$ Given that coverage is mandated for Inrebiq, Impact Biomedicine, the manufacturer, had little to no incentive to be competitive with its launch price or to enter into clinically meaningful value-based contracts with health plans. There are few tools that health plans can use to lower prices in these situations, ultimately increasing costs and premiums across the system.

The preceding issues highlight the greater need for collaboration between the FDA and the Centers for Medicare \& Medicaid Services (CMS), ensuring that FDA drug approval and Part B and Part D coverage is appropriately and consistently reviewed. Ensuring affordability and accessibility to treatments for patients requires new partnerships and facilitation of innovative health insurance and pharmacy benefit designs. Unfortunately, there is currently discordance in policies and timelines between the speed of FDA approvals, the Medicare bid and formulary planning timeline, and coverage mechanisms that account for this rapidly evolving environment of high-cost drug therapies. Although several of the FDA-expedited drug approvals occur in as little as 6 months, the 18 month-bid development timeline in the Medicare Advantage program does not guarantee adequate payment mechanisms for high-cost, new-tomarket drugs approved after bid submission. In addition, uncertainties around launch price and lack of insight into FDA labeling requirements or scope of indicated use until very late in the approval process makes formulary development for Medicare plans a challenging process.

When expedited approval is used to ensure early market access, it is imperative for the health care ecosystem to embrace alternative coverage and financing mechanisms that help mitigate the cost impact of new-to-market drugs, tying reimbursement to effectiveness while also achieving the ultimate goal of addressing unmet medical need.

There are several transitional financing policies already in place for Part B and Part D that could work in tandem to address issues accompanying expedited approval and the pipeline of high-cost drugs, including the National Coverage Determination (NCD), carve out to Medicare feefor-service (FFS), and Coverage with Evidence Development (CED) processes. ${ }^{12-14} \mathrm{CMS}$ could work with the FDA earlier in the approval process to initiate an NCD when a high-cost, high unmet need drug is identified as a candidate for an expedited designation. At this time, if a Part B drug meets the criteria for "significant cost," CMS could also carve out the cost of the drug to the Medicare FFS program to allow Medicare Advantage plans enough time to appropriately account for the cost of the drug in their bid development and understand utilization patterns in their patient populations. Carving out the cost of the drug to FFS would also allow time for a CED process, as part of an NCD, to be initiated and studied.

While an NCD would allow for access at the time of the drug's launch for all beneficiaries throughout the Medicare FFS and Medicare Advantage programs, a CED would also allow for further development of the drug's evidence, informing future payment and coverage decisions in the Medicare Advantage program that are based on sound clinical rationale. It is important to remember that drug manufacturers alone set list prices for prescription drugs. That being the case, during the transitional period in which a drug that received expedited approval is new to the market, common sense would dictate that drug manufactures should bear greater liability, limiting their financial gains until there is demonstration of clinically meaningful outcomes and actual savings to the health care system is realized.

CMS and Congress should help facilitate regulatory policies that empower health plans to execute clinically meaningful value-based contracts with drug manufactures based on quality and value, especially for drugs with limited or no competition. As a trade-off for receiving expedited approval, manufacturers should be required to enter into 
value-based contracts either directly with CMS or Medicare Advantage/ Prescription Drug Plan sponsors. Along with requirements for valuebased contracts, policymakers should consider limiting protected class status and other coverage mandates for drugs with limited competition until appropriate evidence has been developed.

For drugs that receive expedited approval, the FDA should also be conservative with initial labeling requirements, especially when approval is based on surrogate or intermediate endpoints or when the drug has not been fully vetted in terms of safety and efficacy in the clinical trial process, allowing for further label expansion when realworld effectiveness is demonstrated. This point is especially critical within the confines of the Medicare population, which comprises many high-risk, elderly individuals.

These policies will help to further advocate for the de-adoption of lowvalue treatment based on real-world evidence, pending adoption on clinical outcomes, rather than regulatory red tape. The payment and coverage mechanisms previously described bridge real-world clinical effectiveness to payment models, while, most importantly, allowing patients seeking treatment to have appropriate access to potentially life-saving medications.

\section{DISCLOSURES}

No funding supported this commentary. The authors are employed by Humana, Inc. Shrank reports board of directors work for GetWell Network and NCQA. The other authors have nothing to disclose.

\section{REFERENCES}

1. US Food and Drug Administration. Guidance for industry: expedited programs for serious conditions-drugs and biologics. May 2014. Accessed April 12, 2021. https://www.fda.gov/media/86377/ download

2. US Food and Drug Administration. Advancing health through innovation: 2018 new drug therapy approvals. January 2019. Accessed April 12, 2021. https:// www.fda.gov/files/drugs/published/ New-Drug-Therapy-Approvals-2018_3.pdf

3. US Food and Drug Administration. New drug therapy approvals 2019. January 21, 2020. Accessed April 12, 2021. https:// www.fda.gov/drugs/new-drugs-fdacders-new-molecular-entities-andnew-therapeutic-biological-products/ new-drug-therapy-approvals-2020

4. Hampson G, Towse A, Pearson EA. Gene therapy: evidence, value and affordability in the US health care system. J Comp Eff Res. 2018;7(1):15-28. doi: 10.2217/ cer-2017-0068

5. Andrews M. Staggering prices slow insurers' coverage of CAR-T cancer therapy. Kaiser Health News. July 17, 2018. November 13, 2020. https://khn.org/ news/staggering-prices-slow-insurerscoverage-of-car-t-cancer-therapy/

6. Novartis. AveXis announces innovative Zolgensma ${ }^{\circledR}$ gene therapy access programs for US payers and families. May 24, 2019. November 13, 2020. https://www. novartis.com/news/media-releases/ avexis-announces-innovative-zolgensmagene-therapy-access-programs-us-payers-and-families

7. Express Scripts. 2019 drug trend report. 2020. Accessed April 12, 2021. https:// www.express-scripts.com/corporate/ drug-trend-report-2019\#2019-in-review
8. IQVIA Institute. The changing landscape of research and development. April 23, 2019. Accessed April 12, 2021. https://www.iqvia.com/ insights/the-iqvia-institute/reports/ the-changing-landscape-of-researchand-development

9. US Food and Drug Adminstration. Olaratumab (Laratruvo). October 20, 2016. November 13, 2020. https:// www.fda.gov/drugs/resourcesinformation-approved-drugs/ olaratumab-lartruvo

10. Inrebic. Prescribing information. Celgene Corporation; August 2019. Accessed April 12, 2021. https://www. accessdata.fda.gov/drugsatfda docs/ label/2019/212327s000lbl.pdf

11. Jakafi. Prescribing information. Incyte Corporation; May 2019. Accessed April 12, 2021. https://www. accessdata.fda.gov/drugsatfda_docs/ label/2019/202192s017lbl.pdf

12. Centers for Medicare \& Medicaid Services. Medicare Coverage Determination Process. Updated February 23, 2021. Accessed April 12, 2021. https:// www.cms.gov/Medicare/Coverage/ DeterminationProcess

13. Centers for Medicare \& Medicaid Services. Medicare Managed Care Manual. Chapter 8 - payments to Medicare Advantage organizations. Revised September 19, 2014. November 13, 2020. https://www.cms.gov/Regulations-anduidance/Guidance/Manuals/Downloads/ $\underline{\text { mc86c08.pdf }}$

14. Centers for Medicare \& Medicaid Services. Guidance for the public, industry, and CMS staff: coverage with evidence development. November 20, 2014. November 13, 2020. https://www. cms.gov/medicare-coverage-database/ details/medicare-coverage-documentdetails.aspx?MCDId=27 